Pacific Journal of Mathematics

SUPERCOMPLETE SPACES

January 1962 


\section{SUPERCOMPLETE SPACES}

\section{J. R. ISBELL}

A uniform space is supercomplete if its space of closed subsets is complete. The purpose of this note is to characterize the supercomplete spaces, a surprisingly small class, though it includes all complete metric spaces. A uniformisable space admits a supercomplete uniformity if and only if it is paracompact.

The idea and the name of supercomplete spaces were invented by $\mathrm{S}$. Ginsburg and me in 1954. What is new is the simple definition given above, together with the application (of the metric case) in the following paper [5]. Ginsburg and I had an application, a proof that every locally fine uniformity finer than a complete metric uniformity is fine; but the proof which we published [4] is simpler.

If $\mathscr{U}$ is a uniform covering of a uniform space $\mu X$, two subsets $A$ and $B$ of $X$ are said to be within $\mathscr{U}$ of each other if $S t(A, \mathscr{C}) \supset B$ and $S t(B, \mathscr{U}) \supset A$. The set of all closed subsets of $\mu X$ is made into a uniform space $H(\mu X)$ by taking for a basis of uniform coverings the coverings $\{W(A, \mathscr{U}): A \in H(\mu X)\}$, where $W(A, \mathscr{U})$ is the set of all closed subsets of $\mu X$ within $\mathscr{U}$ of $A$ and $\mathscr{U}$ runs through $\mu$.

As was said above, $\mu X$ is called supercomplete when $H(\mu X)$ is complete. At this point we could give a short direct proof that every complete metric space is supercomplete, and go on to the next paper. But to characterize the supercomplete spaces, the following preliminaries seem to be essential.

Recall from [4] that a locally fine space is one in which every uniformly locally uniform covering is uniform; that for every uniform space $\mu X$ there is a next finer locally fine uniformity $\lambda \mu$; that $\lambda \mu$ may be gotten by transfinite iteration of the passage $\mu \rightarrow \mu^{1}$ from the family $\mu$ of uniform coverings to the family $\mu^{1}$ of the uniformly locally uniform coverings; and that the intermediate constructs $\mu^{1}, \mu^{(2)}, \cdots, \mu^{(\alpha)}, \cdots$, while they are not known to be uniformities in the usual sense, are known to be uniformities in the weaker sense of [6].

A function $f$ on a partially ordered set $P$ to a uniform space $\mu X$ is said to be convergent [3] if for every uniform covering $\left\{U_{\alpha}\right\}$ of $\mu X$ there exists a family of residual sets $S_{\alpha}$ in $P$ whose union is cofinal in $P$, such that $f\left(S_{\alpha}\right) \subset U_{\alpha}$ for each $\alpha$. A point $x$ of $X$ is a cluster point of $f$ if for every neighborhood $U$ of $x$ there is a nonempty residual set $S \subset f^{-1}(U)$.

A filter $\mathscr{F}$ of subsets of a uniform space $\mu X$ is said to converge

Received March 27, 1961, An announcement of most of this was published in $[\mathbf{1} ; 115-$ $116]$. 
to $a$ set if the closures of the elements of $\mathscr{F}$ form a convergent net (ordered by inclusion) in $H(\mu X)$. Equivalently, if $K$ is the set of all cluster points of the filter $\mathscr{F}$, every uniform neighborhood of $K$ must contain an element of $\mathscr{F} . \mathscr{F}$ is stable if for every uniform covering $\mathscr{U}_{\ell}$ there is $A \in \mathscr{F}$ such that for every $B \in \mathscr{F}, S t\left(B, \mathscr{Q}_{\ell}\right) \supset A$.

Evidently every filter converging to a set is stable. The converse is obvious for supercomplete spaces. It characterizes supercomplete spaces; and the theorem is

THEOREM. The following conditions on a uniform space $\mu X$ are equivalent :

(a) $\mu X$ is supercomplete;

(b) $X$ is paracompact and $\lambda \mu X$ is fine;

(c) every convergent function with values in $\mu X$ has a cluster point ;

(d) every stable filter in $\mu X$ converges to a set.

Proof. (a) $\Rightarrow($ b). It is equivalent to (b) to say that every open covering of $X$ is in $\lambda \mu$. Suppose this is not the case, $\mathscr{U} \notin \lambda \mu$. We construct a net $N$ of points of $H(\mu X)$, ordered by inclusion; $N$ consists of all those closed $S \subset X$ such that on some uniform neighborhood of $X-S, \mathscr{C}$ coincides with some covering in $\lambda \mu$. Because of the requirement of a uniform neighborhood, $N$ is directed by inclusion. Moreover, $N$ is Cauchy. To prove this we must show that for every $\mathscr{Y}$ in $\mu$, there is $S$ in $N$ such that every $T \subset S$ in $N$ is within $\mathscr{Y}$ of $S$. Let $S$ be the closure of the union of all $V$ in $\mathscr{V}$ such that $\mathscr{U}$ does not coincide on $V$ with any covering in $\lambda \mu . S$ is in $N$ because the union of all other $V$ in $\mathscr{V}^{-}$is a uniform neighborhood $W$ of $X-S$ (it contains $S t(X-S, \mathscr{Y}))$; and on any intermediate uniform neighborhood $Z$ of $X-S$ such that $S t(X-S, \mathscr{Y})$ is a uniform neighborhood of $Z$, $\mathscr{C}$ coincides uniformly locally with coverings in $\lambda \mu$ and therefore coincides with a covering in $\lambda \mu$. Next, for any $T \in N$, every point $x$ of $S$ is in the closure of $S t(T, \mathscr{V})$. Before proving this we note that it will show that if $T \subset S$ then $T$ is within $\mathscr{V}^{*}$ of $S$; since every covering in $\mu$ is refined by some $\mathscr{V}^{*}, \mathscr{V}^{*}$ in $\mu$, this suffices to prove that $N$ is Cauchy. Now $x \in S$ is certainly arbitrarily near to sets $V$ on which $\mathscr{U}$ does not coincide with any covering in $\lambda \mu$; but $\mathscr{U}$ does do this on $X-T$, which means that $X-T$ contains no such set $V$ and $T$ meets all of them.

It remains to note that $N$ is not convergent; in fact, the filter base $N$ has no cluster points, since every point has a neighborhood on which $\mathscr{U}$ is uniform.

(b) $\Rightarrow(c)$. Suppose $\mu X$ satisfies (b), and $f: P \rightarrow \mu X$ is convergent. We show next that $f: P \rightarrow \lambda \mu X$ is also convergent, applying transfinite 
induction on the Morita uniform spaces $\mu^{(\alpha)} X$. Suppose $f: P \rightarrow \mu^{(\alpha)} X$ is nonconvergent for some $\alpha$, and pick the least such $\alpha$. It cannot be a limit ordinal, for then each uniform covering of $\mu^{(\alpha)} X$ is in $\mu^{(\beta)}$ for some $\beta<\alpha$. Hence $\alpha$ must be of the form $\beta+1$; and $\mu^{(\alpha)}$ has a basis consisting of coverings of the form $\left\{U_{i} \cap V_{j}^{i}\right\}$, where $\left\{U_{i}\right\}$ and each $\mathscr{V}^{i}=\left\{V_{j}^{i}\right\}$ are in $\mu^{(\beta)}$. We must show that every $p$ in $P$ has a successor in a residual set $Q$ such that $f(Q) \subset U_{i} \cap V_{j}^{i}$ for some $i$ and $j$. For this, $p$ has a successor $r$ in a residual set mapped into some $U_{i}$; and $r$ has a successor $q$ in a residual set mapped into some $V_{\jmath}^{i}$. Let $Q$ be the set of successors of $q$.

Now if $f$ had no cluster point, there would be an open covering consisting of sets whose inverse images contain no nonempty residual sets. Since every open covering is in $\lambda \mu$, this is absurd.

$(c) \Rightarrow(d)$. Suppose $\mathscr{F}$ is a stable filter in $\mu X$ which fails to converge. Thus the set $K$ of cluster points of $\mathscr{F}$ has a uniform neighborhood $U$ which fails to contain any element of $\mathscr{F}$. Let $P$ be the partially ordered set of all subsets of $X-U$ which contain a uniform neighborhood of a set compatible with $\mathscr{F}$ (meeting every element of $\mathscr{F})$; order $P$ by reverse inclusion. Let $f: P \rightarrow \mu X$ be any choice function. Obviously $f$ has no cluster point; but we shall see that $f$ is convergent. Let $\left\{U_{\alpha}\right\}$ be any uniform covering of $\mu X$. For any $S \in P$, let $S$ be a uniform neighborhood of compatible $T$, and let $\mathscr{Y}$ be a uniform covering so fine that $S t\left(T, \mathscr{Y}^{*}\right) \subset S$ and $\mathscr{Y}^{* *}$ is a refinement of $\left\{U_{\alpha}\right\}$. In the stable filter $\mathscr{F}$ let $A$ be a member which is contained in every $\operatorname{St}\left(B, \mathscr{Y}^{\prime}\right), B \in \mathscr{F}$. Let $x$ be a point of $T \cap A$. Some $U_{a}$ contains $R=S t\left(x, \mathscr{y}^{*}\right)$, which is a subset of $S$ also; moreover, since $x \in A$, every $B$ in $\mathscr{F}$ meets $S t(x, \mathscr{V})$. Thus $R$ is a uniform neighborhood of a compatible set, and $R$ is a successor of $S$ all of whose successors are mapped into $U_{\alpha}$ by $f$.

The proof that (d) implies (a) amounts to no more than verifying that if $\left\{S_{\lambda}\right\}$ is a Cauchy net in $H(\mu X)$ then the sets which contain almost all $S_{\lambda}$ form a stable filter; and we omit it.

COROLlary. Every complete metric space is supercomplete.

\section{Proof. It satisfies (b) [4].}

Finally we note the connection with H. H. Corson's weakly Cauchy filters, which have cluster points in an undetermined class of paracompact uniform spaces including the fine paracompact spaces [2]. A stable filter is weakly Cauchy. Since we exhibited above (in (a) $\Rightarrow(b)$ ) a stable filter without cluster points in an arbitrary nonsupercomplete space, Corson's class is a subclass of the supercomplete spaces. Corson and I satisfied ourselves in 1958 that it is a proper subclass. 


\section{REFERENCES}

1. R. Bing, ed., Summary of lectures and seminars, Summer Institute on Set Theoretic Topology, Madison, 1955.

2. H. Corson, The determination of paracompactness by uniformities, Amer. J. Math., 80 (1958), 185-190.

3. S. Ginsburg and J. Isbell, Rings of convergent functions (Abstract), Bull. Amer. Math. Soc., 60 (1954), 259.

4. - Some operators on uniform spaces, Trans. Amer. Math. Soc., 93 (1959), $145-168$.

5. J. Isbell, On finite-dimensional uniform spaces, II, Pacific J. Math., 12 (1962), 291-302.

6. K. Morita, On the simple extension of a space with respect to a uniformity, I, Proc. Japan Acad., 27 (1951), 65-72.

UNIVERSITY OF WASHINGTON 


\section{PACIFIC JOURNAL OF MATHEMATICS}

\section{EDITORS}

Ralph S. Phillips

Stanford University

Stanford, California

M. G. Arsove

University of Washington

Seattle 5, Washington
A. L. Whiteman

University of Southern Californla

Los Angeles 7, California

Lowell J. Paige

University of California

Los Angeles 24, California

\section{ASSOCIATE EDITORS}

E. F. BECKENBACH

D. DERRY

H. L. ROYDEN

E. G. STRAUS

T. M. CHERRY

M. OHTSUKA

E. SPANIER

F. WOLF

\section{SUPPORTING INSTITUTIONS}

UNIVERSITY OF BRITISH COLUMBIA

STANFORD UNIVERSITY

CALIFORNIA INSTITUTE OF TECHNOLOGY

UNIVERSITY OF CALIFORNIA

MONTANA STATE UNIVERSITY

UNIVERSITY OF TOKYO

UNIVERSITY OF UTAH

UNIVERSITY OF NEVADA

NEW MEXICO STATE UNIVERSITY

OREGON STATE UNIVERSITY

UNIVERSITY OF OREGON

OSAKA UNIVERSITY

WASHINGTON STATE UNIVERSITY

UNIVERSITY OF WASHINGTON

UNIVERSITY OF SOUTHERN CALIFORNIA

AMERICAN MATHEMATICAL SOCIETY CALIFORNIA RESEARCH CORPORATION SPACE TECHNOLOGY LABORATORIES NAVAL ORDNANCE TEST STATION 


\section{Pacific Journal of Mathematics}

\section{Vol. 12, No. $1 \quad$ January, 1962}

Jonathan L. Alperin, Groups with finitely many automorphisms $\ldots \ldots \ldots \ldots \ldots \ldots \ldots \ldots$

Martin Arthur Arkowitz, The generalized Whitehead product ................ 7

John D. Baum, Instability and asymptoticity in toplogical dynamics . . . . . . . . . . 25

William Aaron Beyer, Hausdorff dimension of level sets of some Rademacher series .... $\quad 35$

Frank Herbert Brownell, III, A note on Cook's wave-matrix theorem . . . . . . . . . . . . . 47

Gulbank D. Chakerian, An inequality for closed space curves ................. 53

Inge Futtrup Christensen, Some further extensions of a theorem of Marcinkiewicz ....... 59

Charles Vernon Coffman, Linear differential equations on cones in Banach spaces . . . . . 69

Eckford Cohen, Arithmetical notes. III. Certain equally distributed sets of integers . . . . . 77

John Irving Derr and Angus E. Taylor, Operators of meromorphic type with multiple poles

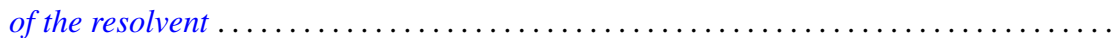

Jacob Feldman, On measurability of stochastic processes in products space .............

Robert S. Freeman, Closed extensions of the Laplace operator determined by a general class of boundary conditions, for unbounded regions ......................

Robert E. Fullerton, Geometric structure of absolute basis systems in a linear topological

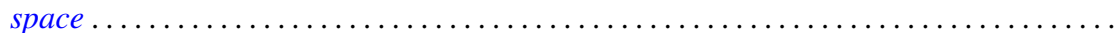

Dieter Gaier, On conformal mapping of nearly circular regions

Andrew Mattei Gleason and Hassler Whitney, The extension of linear functionals defined

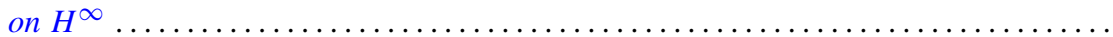

Seymour Goldberg, Closed linear operators and associated continuous linear

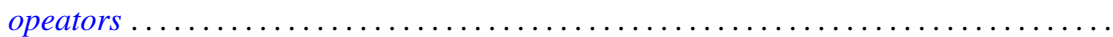

Basil Gordon, Aviezri Siegmund Fraenkel and Ernst Gabor Straus, On the determination of sets by the sets of sums of a certain order

Branko Grünbaum, The dimension of intersections of convex sets. .

Paul Daniel Hill, On the number of pure subgroups

Robert Peter Holten, Generalized Goursat problem . .

Alfred Horn, Eigenvalues of sums of Hermitian matrices ...........

Henry C. Howard, Oscillation and nonoscillation criteria for

$$
y^{\prime \prime}(x)+f(y(x)) p(x)=0
$$

Taqdir Husain, $S$-spaces and the open mapping theorem ...

Richard Eugene Isaac, Markov processes and unique stationary probability measures ...

John Rolfe Isbell, Supercomplete spaces ....................

John Rolfe Isbell, On finite-dimensional uniform spaces. II .........

N. Jacobson, A note on automorphisms of Lie algebras ..............

Antoni A. Kosinski, A theorem on families of acyclic sets and its applications

Marvin David Marcus and H. Minc, The invariance of symmetric functions of singular values...

Ralph David McWilliams, A note on weak sequential convergence.

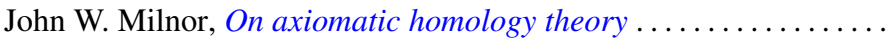

Victor Julius Mizel and Malempati Madhusudana Rao, Nonsymmetric projections in

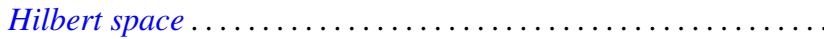

Calvin Cooper Moore, On the Frobenius reciprocity theorem for locally compact

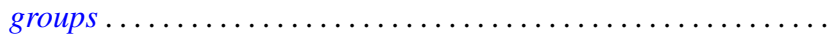

Donald J. Newman, The Gibbs phenomenon for Hausdorff means . 